\title{
PHOTONIC CRYSTAL WAVEGUIDE BIOSENSOR
}

\author{
A. A. ZANISHEVSKAYA \\ Saratov State University \\ 83 Astrakhanskaya Str., Saratov 410012, Russia \\ zan-anastasiya@yandex.ru \\ A. V. MALININ \\ Saratov State University \\ 83 Astrakhanskaya Str., Saratov 410012, Russia \\ SPE Nanostructured Glass Technology \\ 101, 50 let Oktjabrja ave., Saratov 410033, Russia \\ V. V. TUCHIN \\ Saratov State University \\ 83 Astrakhanskaya Str., Saratov 410012, Russia \\ Institute of Precise Mechanics and Control RAS \\ 24, Rabochaya Str., Saratov 410028, Russia \\ University of Oulu, P. O. Box 4500, Oulu FIN-90014, Finland \\ YU. S. SKIBINA \\ Saratov State University \\ 83 Astrakhanskaya Str., Saratov 410012, Russia \\ SPE Nanostructured Glass Technology \\ 101, 50 let Oktjabrja Ave., Saratov 410033, Russia \\ I. YU. SILOKHIN \\ SPE Nanostructured Glass Technology \\ 101, 50 let Oktjabrja Ave., Saratov 410033, Russia
}

Received 29 October 2012

Accepted 30 December 2012

Published 5 March 2013

\begin{abstract}
The hollow core photonic crystal waveguide biosensor is designed and described. The biosensor was tested in experiments for artificial sweetener identification in drinks. The photonic crystal waveguide biosensor has a high sensitivity to the optical properties of liquids filling up the hollow core. The compactness, good integration ability to different optical systems and compatibility for use in industrial settings make such biosensor very promising for various biomedical applications.
\end{abstract}

This is an Open Access article published by World Scientific Publishing Company. It is distributed under the terms of the Creative Commons Attribution 3.0 (CC-BY) License. Further distribution of this work is permitted, provided the original work is properly cited. 


\section{A. A. Zanishevskaya et al.}

Keywords: Biosensor; hollow core photonic crystal fiber; biomedical applications; artificial sweetener; food quality.

\section{Introduction}

Last decade brought photonic crystal waveguides (PCW) to be used in fiber optic and micro-optic spectroscopy-based devices designed for biology and medicine. ${ }^{1} \mathrm{PCW}$ is a kind of optical fiber that presents a diversity of new and improved features beyond that conventional optical fiber can offer. ${ }^{2}$ In 2008, the hollow core PCW with chirped cladding were first designed and investigated by our group. ${ }^{3}$ A number of PCW multifunctional optical sensors and sample collectors as well as their biomedical applications for glucose, vitamin sensing and blood type identification were recently demonstrated..$^{1,3-7}$ The basis for these applications is a wide spectral range response of this type of biosensor to changes of index of refraction, absorption and scattering properties of the probed medium inside the sensor. The goal of this paper is to demonstrate further biosensing abilities of holey PCWs with chirped cladding. As objects for testing we took juices with natural and artificial sugars in order to show biosensor sensitivity to small changes in index of refraction of a multicomponent liquid. This study has not only methodological goal but instead the data received can also be directly used in food industry to provide healthy and controllable beverage production, especially for diabetic population. Since artificial sweeteners appeared on the market in the 1980s, the possible health risks from these substances have been discussed. Researchers have linked the sweeteners with diabetes, fibromyalgia, certain forms of cancer and many other health problems including headaches, seizures and brain tumors. ${ }^{8,9}$ Artificial sweeteners can be found in many products, including drinks, yoghurts, sweets and medications. The safety or harm of many artificial sweeteners has not yet been proved, but the manufacturers should keep consumers informed about the presence of sweeteners in the foodstuff for fair. The sweetness of juices or other drinks depends on the content of different sugars (glucose, fructose), artificial sweeteners, sugar substitutes or their different combinations. In many cases using artificial sweeteners instead of natural sugar, like glucose or fructose, are more preferable because of their low cost. In some cases, dishonest manufacturers conceal this fact so the search for simple and automated methods for monitoring of drinks quality is of great importance.

Evidently, further biomedical applications of the designed PCW biosensor for analysis of human bioliquids and their components can be provided using results of this study.

\section{Methods}

\subsection{PCW biosensor}

The basic element of designed biosensor is the PCW with the structured cladding representing the twodimensional photonic crystal which is formed by layers of longitudinal holes arranged in correspondence with a certain law (see Fig. 1). This type of PCW, which enables interaction of the liquid analyte with fundamental optical modes, propagating through the fiber's core, has a great potential to become a basis for highly sensitive optical analyzer.

The broadband optical radiation (see Fig. 2) propagated through the PCW changes appreciably its spectral parameters: the output radiation signature contains explicit photonic band gaps (see Fig. 3) which location depends on the fiber cladding features and spectral/dispersive properties of the medium, filling up the internal channels of the PCW.

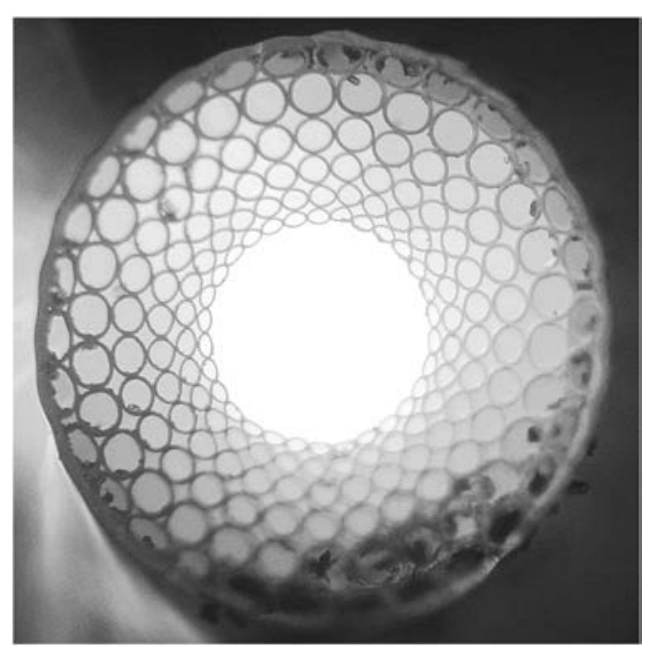

Fig. 1. The microphotograph of the cross-section of the hollow core $\mathrm{PCW}$. 


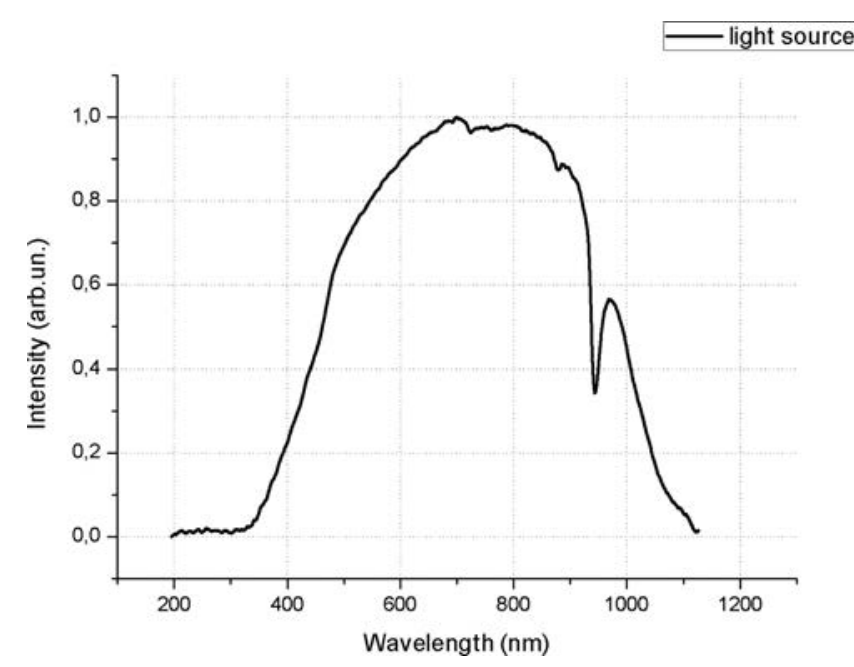

Fig. 2. The spectrum of the light source.

The response of the PCW output spectral characteristics to the filled up medium (gas or liquid) properties, such as absorption, index of refraction and scattering, determines the promising applications of the waveguides in the analytic devices (sensors). The PCW application for sensor design was proposed first in Ref. 3, where the absorption spectra of acetylene that filled up a waveguide under pressure were measured. The photonic crystal sensor that allows for detection of individual molecules via their two-photon fluorescence was described. ${ }^{1}$ The selective detection of antibodies in a PCW was also realized. ${ }^{1,6}$

Recent experiments provided by our research group $^{1,6,7}$ revealed that position of photonic bandgap of a hollow PCW are largely affected by the refractive index $(\mathrm{RI})$ of the medium, filling the space

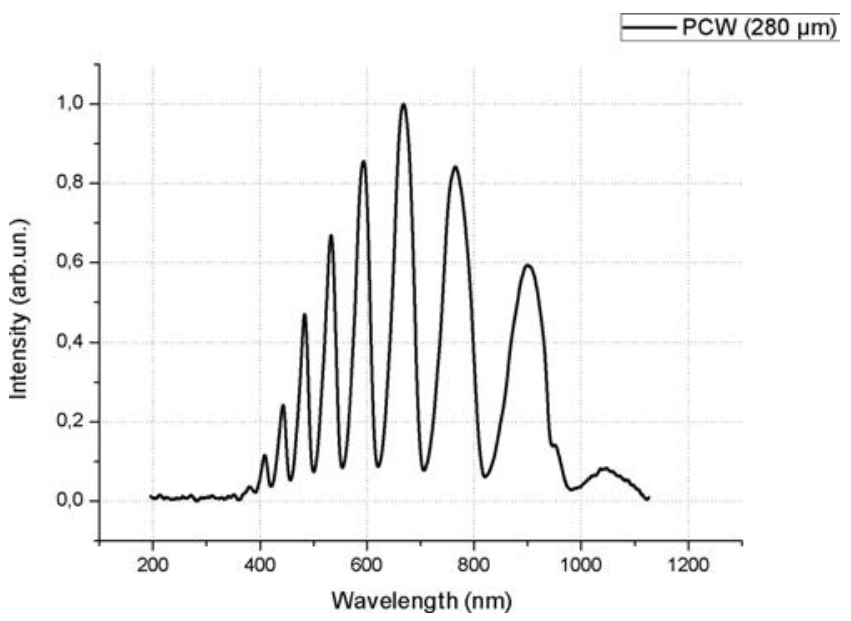

Fig. 3. Transmission spectrum of the chirped photonic crystal waveguide (hollow core diameter is $280 \mu \mathrm{m}$ ). in the hollow core and the channels of the cladding. The RI change induces a predictable transformation of PCW transmission spectrum. ${ }^{1,6,7}$ The RI $n_{c}$ of a medium inside a hollow core determines the wavelengths of high transmission of the optical radiation through the waveguide. Transmission bands are shifting to shorter wavelengths as RI increases.

The use of PCW as an optical biosensor allows one to get an instant optical response to the change of RI of the analyzed medium. Further, we will consider the possibility of using PCW with a hollow core as an optical sensor for juice quality analysis, more specifically, for the determination of presence of sweeteners inside.

\subsection{Sweetness of the drinks}

One of the most important parameters for juice or wine qualification is a sugar fraction evaluation and detection of additional sweeteners. The average sugar content in apple juice, for example, is $7-12 \mathrm{~g} /$ $100 \mathrm{~mL} .{ }^{10}$ The widely used technique for sugar determination in food industry is a single wavelength refractometric method which based on the measurement of RI of the liquid and calculation of sugar amount using the following linear expression:

$$
n=n_{w}+a C,
$$

where $n_{w}$ is the $\mathrm{RI}$ of water $\left(25^{\circ} \mathrm{C}\right), a$ is the specific increment of a refraction being equal to 0.00143 and $C$ is the concentration of sugar in $\mathrm{g} / 100 \mathrm{~mL}$.

During the process of fruit juices production, different synthetic sweeteners can be added to drinks in order to improve its taste. Such sweeteners are in 10 and 100 times sweeter than glucose, fructose and sucrose. Nevertheless, none of sweeteners have the same organoleptic qualities as a natural sugar; therefore different mixtures of sweeteners are mostly used for drinks production. This is because it has been found that they have a synergistic effect whereby the sweetness of a mixture is greater than that of the individual components. This means less can be used to get the same apparent sweetness. One of the most popular and cost-saving sweeteners is a mixture of cyclamate (E952) and saccharin (E954). It is 120-210 times as sweeter than sugar and it is not metabolized and thus noncaloric.

Required amount of the sweetener can be calculated using formula:

$$
M=C / K_{\mathrm{sw}},
$$




\section{A. A. Zanishevskaya et al.}

where $C$ is the amount of substitutable sugar, $M$ is the required amount of sweetener and $K_{\mathrm{sw}}$ is the coefficient of sweetness.

Obviously, the solution with artificial sweeteners has a low RI than for natural sugar because of its low concentration. In fact the RI of $7 \mathrm{~g} / 100 \mathrm{~mL}$ glucose solution is $1.3534\left(t=25^{\circ} \mathrm{C}\right)$, but the concentration of the sweetener solution with the same sweetness degree is only $0.058 \mathrm{~g} / 100 \mathrm{~mL}$ and its RI is $1.3329\left(t=25^{\circ} \mathrm{C}\right)$. In our experiments, we are detecting the difference in spectral characteristics of PCFs samples, filled up with liquids, which contains natural sugar and the mixture of sweeteners.

\section{Results}

The aim of the first stage of the study was to detect the spectral responses for injection of liquids, containing natural sugar (glucose) and the mixture of sweeteners, into PCW internal structure. As a model liquid, we used aqueous solution of glucose with concentration $7 \mathrm{~g} / 100 \mathrm{~mL}$ (average sugar content for an apple juice) and aqueous solution of the cyclamate and saccharin mixture with $0.058 \mathrm{~g} /$ $100 \mathrm{~mL}$ concentration. The PCW sample length was $60 \mathrm{~mm}$, hollow core diameter was $280 \mu \mathrm{m}$, liquid sample volume $-10 \mu \mathrm{L}$. Obtained transmission spectra is plotted in Fig. 4. Comparing two spectra, one can see that due to difference of RI of two solutions, the corresponding transmission bands of the PCW are shifted relative to each other. The higher RI of the glucose solution manifests in a shift of transmission bands to shorter wavelength region.

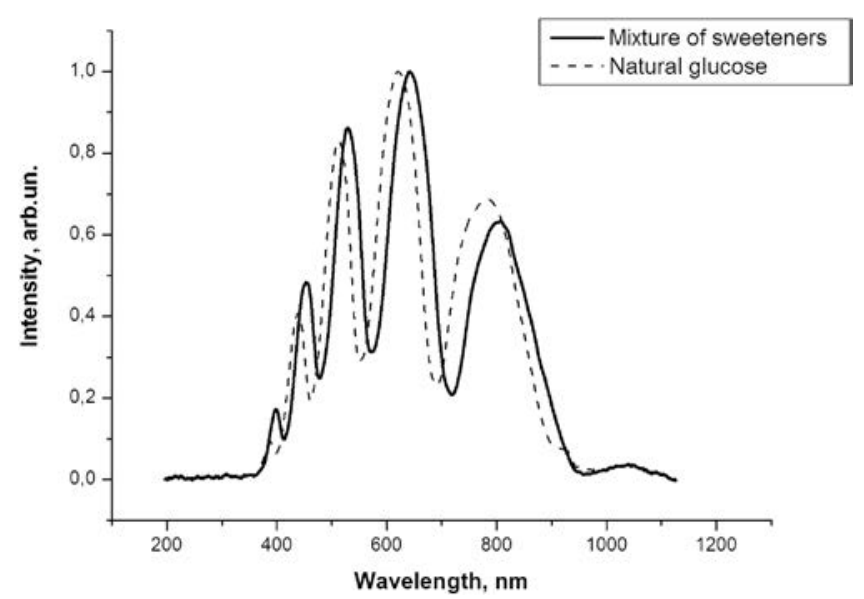

Fig. 4. Transmission spectra of the chirped PCF samples, filled up with aqueous solution of glucose and aqueous solution of cyclamate and saccharin mixture.

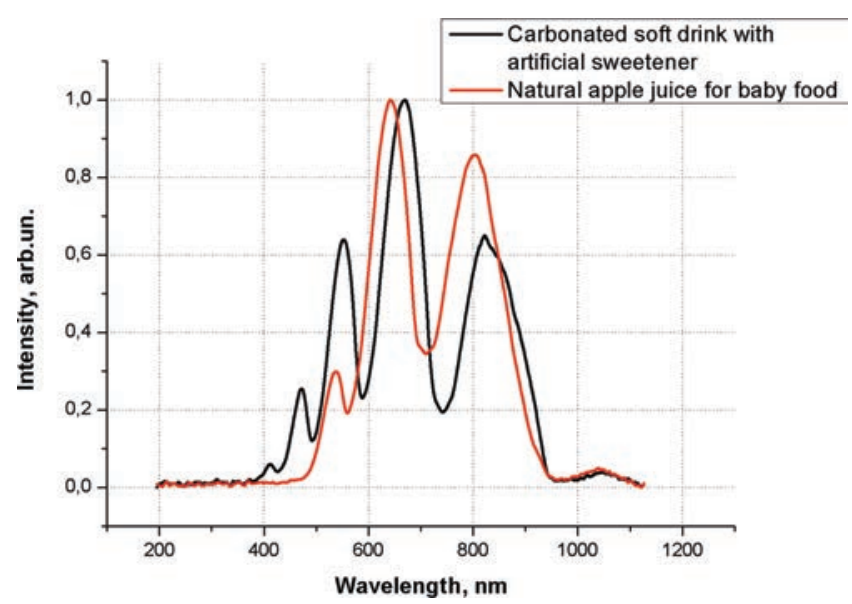

Fig. 5. Normalized transmission spectra of the PCW samples, filled up with natural apple juice for baby food and carbonated soft drink with artificial sweetener.

This shift can be used as an indicator of natural sugar or sweetener substitution.

After calibration of PCW sensor we obtained transmission spectra of the PCW filled up with three samples of drinks: natural apple juice for baby food, carbonated soft drinks with artificial sweetener and with natural sugar.

Captured transmission spectra are plotted in Figs. 5 and 6. Due to slight absorption of light in visible spectral range in these liquids, an attenuation of transmission peaks, which are within the absorption band, appears. Despite the significant attenuation of some transmission maxima, one can see that transmission bands in spectra corresponding to two PCF samples, filled up with natural

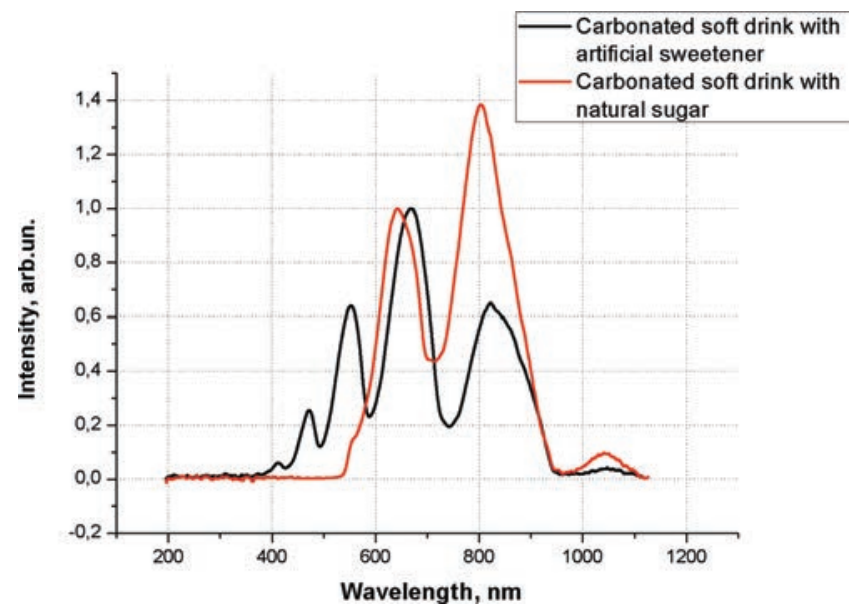

Fig. 6. Normalized transmission spectra of the PCW samples, filled up with carbonated soft drink with artificial sweetener and natural sugar. 


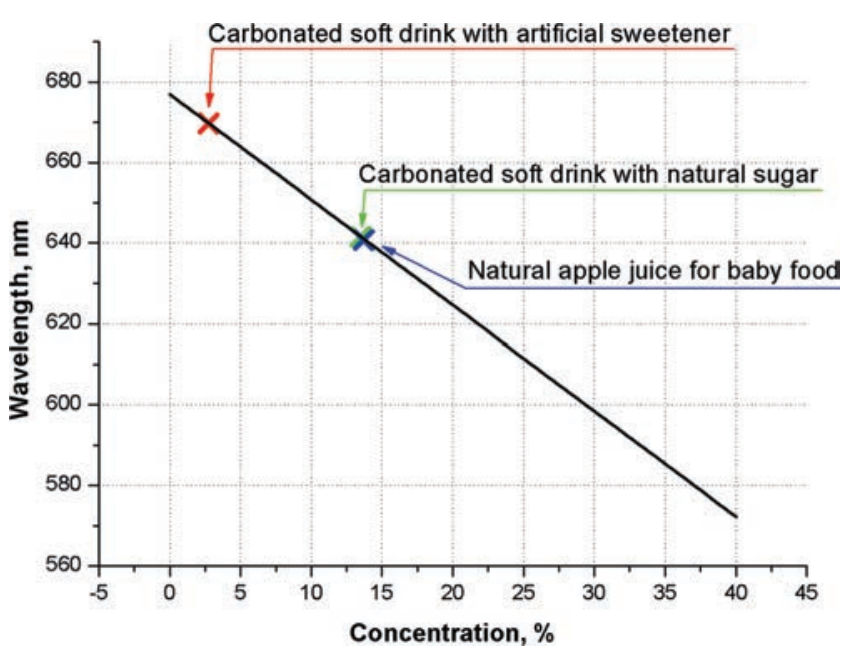

Fig. 7. Dependence for shift of transmission maximum of PCW filled up with glucose solution on its concentration (solid line, calibration) and experimental data for three samples of soft drinks.

apple juice for baby food and carbonated soft drinks with natural sugar, are shifted to the shorter wavelength range relative to transmission band of the sample with carbonated soft drinks with artificial sweetener.

Earlier in Ref. 7 we demonstrated an ability to determine glucose concentration within the range of $1-36 \mathrm{~g} / 100 \mathrm{~mL}$ interval using chirped PCW with big grating period. It was shown that, one can obtain a spectral response to glucose concentration increase in liquid by filling up the internal channels of the PCW with analyzed medium and capturing its transmission spectrum. These measurements allowed us to get the dependence of location of chosen intensity maximum on glucose concentration and use it as a calibration graph to calculate the total sugar concentration. Using this calibration graph we calculated the total natural sugar concentration from the spectra of the three sample

Table 1. Determination of sugar content in different drinks.

\begin{tabular}{ccc}
\hline Drink & $\begin{array}{c}\text { Transmission } \\
\text { peak, nm }\end{array}$ & $\begin{array}{c}\text { Calculated } \\
\text { sugar } \\
\text { concentration, \% }\end{array}$ \\
\hline $\begin{array}{c}\text { Natural apple juice for } \\
\text { baby food }\end{array}$ & 640,8 & 13,7 \\
$\begin{array}{c}\text { Carbonated soft drink } \\
\text { with natural sugar } \\
\begin{array}{c}\text { Carbonated soft drink } \\
\text { with artificial }\end{array}\end{array}$ & 641,5 & 13,5 \\
sweetener & 669,8 & 2,7 \\
\hline
\end{tabular}

drinks (see Fig. 7). The calculated values were presented in Table 1.

\section{Discussion}

Since 1950s, specialists try to apply optical spectroscopy quality and safety controls for the food and beverage industry. Optical-fibers-based sensors are low cost and efficient solutions for this industry due to their high sensitivity, small size and low-cost instrumentation. PCW are, inherently, optical fiber differing from well-known classical silica optical fiber by a number of design features and the principle of light propagation. The diversity of unusual features of PCW leads to an increase of possibilities for new and improved sensors.

The photonic crystal fibers sensing is a field that is growing by the day, and so there are many publications and patents described to this. There are many developed photonic crystal fibers based on sensors: physical sensors, pressures sensors, RI sensors, gas sensors, DNA sensors and many others.

$\mathrm{RI}$ is a fundamental material property. Its accurate measuring is crucial in many applications. In food or beverage industries, the monitoring of RI is a part of the quality control. RI sensor based on solid-core PCW are described in Refs. 11 and 12 and on hollow core PCW in Refs. 4 and 13.

In our investigation, we use a special type of PCWs - chirped PCW with big grating period. The chirped PCWs provide similar transmission properties as the classical PCWs, with one significant difference: they possess a few narrow and smooth transmission bands in the visible range of electromagnetic spectrum. The position of the optical radiation transmission regions of $\mathrm{PCW}$ are largely affected by the RI of the medium, filling the space in the hollow core and the channels of the cladding.

It should be noted that the relevant advantage of using of PCW is the possibility of multi-parameter analysis. By making only one measurement, we can obtain both, optical density value (in determined spectral range) and RI of a tested liquid. This was described in detail in our previous works. ${ }^{1,6,7}$

\section{Conclusion}

Obtained experimental data demonstrates ability for use of PCWs as a "smart" optical element, which gives optical response to the change of injected medium parameters, more specifically to RI 


\section{A. A. Zanishevskaya et al.}

change. In the case of drink quality analysis, RI change is mainly determined by sugar fraction increase (or decrease) in liquid and can be definitely associated with this parameter.

Significant reduction of the sample volume of analyzed liquid is also one of the advantages of use of the photonic crystal fibers as biosensor elements. The use of standard photometric cuvette with $10 \mathrm{~mm}$ thickness requires $2-3 \mathrm{~mL}$ of analyzed liquid for one measurement, while $10-15 \mu \mathrm{L}$ volume is required for filling up PCW sample of $60 \mathrm{~mm}$ length, which also allows for immediate six-fold increase of sensitivity due to bigger light pathway.

\section{Acknowledgments}

This study was partly supported by Grant No. 224014 PHOTONICS4LIFE of FP7-ICT-2007-2; Project No. 1.4.09; RF Governmental contracts 11. 519.11.2035, 14.B37.21.0728 and 14.B37.21.0563; FiDiPro, TEKES Program (40111/11), Finland; SCOPES EC, Uzb/Switz/RF, Swiss NSF, IZ74ZO_ 137423/1; RF President's Grant 1177.2012.2 "Scientific Schools".

\section{References}

1. Yu. S. Skibina, V. V. Tuchin, V. I. Beloglazov, G. Steinmeyer, J. Bethge, R. Wedell, N. Langhoff, "Photonic crystal fibers in biomedical investigations," Quantum Electron. 41(4), 284-301 (2011).

2. P. S. J. Russell, "Photonic crystal fibers," Science 299, 358-362 (2003).

3. J. S. Skibina, R. Iliew, J. Bethge, M. Bock, D. Ficher, V. I. Beloglazov, R. Wedell, G. Scheinmeyer, "A chirped photonic crystal fiber," Nat. Photon. 2, $679-683$ (2008).

4. S. Konorov, A. Zheltikov, M. Scalora, "Photoniccrystal fiber as a multifunctional optical sensor and sample collector," Opt. Express 13(9), 3454-3459 (2005).

5. N. Skivesen, A. Têtu, M. Kristensen, J. Kjems, L. H. Frandsen, P. I. Borel, "Photonic-crystal waveguide biosensor," Opt. Express 15(6), 3169-3176 (2007).

6. A. V. Malinin, Yu. S. Skibina, V. V. Tuchin, M. V. Chainikov, V. I. Beloglazov, I. Yu. Silokhin, A. A. Zanishevskaya, V. A. Dubrovskii, A. A. Dolmashkin, "The use of hollow-core photonic crystal fibres as biological sensors," Quantum Electron. 41(4), 302-307 (2011).

7. A. V. Malinin, A. A. Zanishevskaja, V. V. Tuchin, Yu. S. Skibina, I. Yu. Silokhin, "Oxidase method for glucose determination using long-period grating waveguide," Proc. SPIE 8222, 82221B (2012).

8. M. Soffritti, F. Belpoggi, M. Manservigi, E. Tibaldi, M. Lauriola, L. Falcioni, L. Bua, "Aspartame administered in feed, beginning prenatally through life span, induces cancers of the liver and lung in male Swiss mice," Amer. J. Ind. Med. 53(12), 1197-1206 (2010).

9. J. W. Olney, N. B. Farber, E. Spitznagel, L. N. Robins, "Increasing brain tumor rates: Is there a link to aspartame?," J. Neuropathol. Exp. Neurol. 55(11), 1115-1123 (1996).

10. J. Markowski, A. Baron, M. Mieszczakowska, W. Płocharski, "Chemical composition of French and Polish cloudy apple juices," J. Horticultural Sci. Biotechnol. 84(6), 68-74 (2009).

11. A. M. R. Pinto, M. Lopez-Amo, "Photonic crystal fibers for sensing applications," J. Sensors 2012, 21 (2012).

12. D. K. C. Wu, B. T. Kuhlmey, B. J. Eggleton, "Ultrasensitive photonic crystal fiber refractive index sensor," Opt. Lett. 34(3), 322-324 (2009).

13. L. Rindorf, O. Bang, "Sensitivity of photonic crystal fiber grating sensors: Biosensing, refractive index, strain, and temperature sensing," J. Opt. Soc. Amer. B 25(3), 310-324 (2008). 